\title{
Brief self-efficacy interventions to increase healthy dietary behaviours: evidence from two randomized controlled trials
}

\author{
Emily P. Bouwman, Marleen C. Onwezen and Danny Taufik \\ Department of Wageningen Economic Research, \\ Wageningen University and Research, Wageningen, The Netherlands \\ David de Buisonjé \\ Department of Health, Medical and Neuropsychology, \\ Institute of Psychology, Leiden University, Leiden, The Netherlands, and \\ Amber Ronteltap \\ Knowledge Centre Healthy and Sustainable Living, \\ University of Applied Sciences Utrecht, Utrecht, The Netherlands
}

\begin{abstract}
Purpose - Self-efficacy has often been found to play a significant role in healthy dietary behaviours. However, self-efficacy interventions most often consist of intensive interventions. The authors aim to provide more insight into the effect of brief self-efficacy interventions on healthy dietary behaviours.

Design/methodology/approach - In the present article, two randomized controlled trials are described. In study 1, a brief self-efficacy intervention with multiple self-efficacy techniques integrated on a flyer is tested, and in study 2 , an online brief self-efficacy intervention with a single self-efficacy technique is tested.

Findings - The results show that a brief self-efficacy intervention can directly increase vegetable intake and indirectly improve compliance to a diet plan to eat healthier.

Originality/value - These findings suggest that self-efficacy interventions do not always have to be intensive to change dietary behaviours and that brief self-efficacy interventions can also lead to more healthy dietary behaviours.
\end{abstract}

Keywords Brief intervention, Healthy dietary behaviour, Fruit and vegetable intake, Self-efficacy,

Randomized controlled trial

Paper type Research paper

\section{Introduction}

According to global estimates of the World Health Organization (2003), worldwide obesity has nearly tripled since 1975. In 2016,39\% of adults aged 18 years and over were overweight and $13 \%$ were obese (Obesity and overweight, 2018). This is largely caused by current eating

(C) Emily P. Bouwman, Marleen C. Onwezen, Danny Taufik, David de Buisonjé and Amber Ronteltap. Published by Emerald Publishing Limited. This article is published under the Creative Commons Attribution (CCBY 4.0) licence. Anyone may reproduce, distribute, translate and create derivative works of this article (for both commercial and non-commercial purposes), subject to full attribution to the original publication and authors. The full terms of this licence may be seen at http://creativecommons. org/licences/by/4.0/legalcode

The authors wish to thank Professor Rob Holland for the contribution with regard to the design of the first study and Dr Harm Veling for the valuable comments provided on the first study. Study 2 was financially supported by the Ministry of Economic Affairs TO2 flex programme. The funder had no role in the design, analysis or writing of this article. The authors wish to thank the research team from the TO2-flex programme, in particular Dr Muriel Verain, Dr Machiel Reinders and Jos van den Puttelaar.

Received 18 July 2019 Revised 28 May 2020 Accepted 28 May 2020

Brief selfefficacy interventions

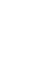


BFJ

122,11

habits, which often consist of too many unhealthy and energy-dense products and too few healthy products. A joint WHO and FAO [1] report (2003) recommends a daily intake of at least $400 \mathrm{~g}$ of fruits and vegetables. However, fruit and vegetable intake is often below recommendations in most affluent countries. Furthermore, most individuals eat too much salt (Hendriksen et al., 2014), saturated fat (Eilander et al., 2015) and calories (Crino et al., 2015). These unhealthy eating habits increase the prevalence of diet-related diseases, such as type 2 diabetes. Therefore, it is important that a shift in dietary behaviours occurs.

Correlational research (Annesi, 2011; Guillaumie et al. 2010; Fernández et al., 2015; Storm et al., 2017) and experimental field studies (e.g. Luszczynska et al., 2007; Kreausukon et al., 2012; Luszczynska et al., 2016) have shown that self-efficacy, the conviction that one can successfully execute a behaviour required to produce certain outcomes (Bandura, 1977), often plays an important role in the performance of healthy dietary behaviours. Over the years, selfefficacy has successfully been used to target a broad range of health topics, such as fruit and vegetable intake. However, the way self-efficacy is currently being targeted is often rather intensive and requires much effort from a participant (Prestwich et al., 2014). For example, Kreausukon et al. (2012) targeted self-efficacy with lectures and a psychological programme, and Luszczynska et al. (2016) and Guillaumie et al. (2012) implemented sessions with group and/or face-to-face components. This is not surprising, since self-efficacy originates from clinical psychology (Bandura, 1977), where behavioural techniques to target self-efficacy often include direct contact between a professional and a patient (Bandura, 1977).

Nevertheless, such intensive interventions require relatively much resources, both for the professional executing the intervention and for the person who is targeted with the intervention. Resources are often scarce. A recent systematic review suggests that in general, brief interventions, which are limited in the number and length of contact moments, can also be effective and improve short-term dietary behaviours (Whatnall et al., 2018). Furthermore, Whatnall et al. (2018) argue that brief interventions can have certain advantages over more intensive interventions when it comes to effectiveness, reach and engagement. For instance, brief interventions are cost-effective due to reduced time and expertise requirements compared with more intensive interventions. Moreover, people might be more inclined to participate in a brief intervention, because it requires limited effort from participants in terms of time and commitment.

However, there is limited evidence for the effectiveness of brief self-efficacy interventions on dietary behaviours; Whatnall et al. (2018) report merely a few studies that use self-efficacy techniques as a brief behavioural intervention to target dietary behaviours. Even though there is little evidence, these few studies do show potential for brief self-efficacy interventions. For instance, Luszczynska et al. (2007) delivered a successful self-efficacy intervention through sending an individually tailored email with information about self-efficacy, feedback with personal results and multiple self-efficacy techniques. Furthermore, Kellar and Abraham (2005) tested a brief intervention that employed persuasive communication targeting selfefficacy, along with other mechanisms. Their intervention did not influence self-efficacy, but did influence intentions, anticipated regret and self-reported fruit and vegetable intake (Kellar and Abraham, 2005). This paper aims to add to this knowledge by examining brief interventions that exclusively focus on self-efficacy techniques, rather than also considering other mechanisms. The current paper will contribute to the understanding of the concept selfefficacy and its range of possibilities in influencing behaviour. Moreover, exploring the potential of brief self-efficacy interventions could promote the use and implementation of selfefficacy strategies and increase healthy dietary behaviours on a larger scale.

H1. A brief self-efficacy intervention can promote healthy dietary behaviours

H2. Self-efficacy positively mediates the effect of a brief self-efficacy intervention on healthy dietary behaviours 
The present studies

The aim of the current studies was to gain more insight into the effects of brief self-efficacy interventions on healthy dietary behaviours and its underlying mechanisms to better understand why these interventions can be effective. Insight into the underlying mechanisms gives valuable information on the success or failure of interventions (O'Rourke and MacKinnon, 2018). In study 1, we investigated whether a brief offline multi-component selfefficacy intervention was successful in increasing healthy dietary behaviours. We used a combination of self-efficacy techniques, as Prestwich et al. (2014) showed that this is more likely to be effective in changing dietary behaviours than use of a single self-efficacy technique. However, an intervention that implements just one technique has the advantage that mediation effects, in our case of self-efficacy, can be more easily detected (Loeys et al., 2015). Therefore in study 2 , we implemented a brief intervention in a more controlled setting (online), while manipulating self-efficacy via a single self-efficacy technique.

\section{Study 1 [2]}

Study 1 aimed to test whether a brief offline multi-component self-efficacy intervention can influence fruit and vegetable intake. Multiple self-efficacy techniques were integrated on a flyer and sent to participants' homes. The outcome variable of the first study was fruit and vegetable intake, for it is below national recommendations (Brink et al., 2016), associated with better health and prevention of diseases (Guillaumie et al., 2012), and self-efficacy has been linked to fruit and vegetable intake in previous studies (e.g. Kreausukon et al., 2012; Luszczynska et al., 2016).

\section{Method}

Participants. Participants were recruited through the "TasteNet-panel" of the FBR institute at Wageningen University and Research Centre in the Netherlands. In total, 1,158 panel members were approached via email. To increase response rate, participants had a chance to win $\mathrm{a} € 40$ voucher and reminders were sent halfway the recruitment phase. In total, 223 participants applied for the study, 211 participants filled in the pre-test, 204 participants received the brief intervention and 198 participants also filled in the post-test. Three participants were excluded from the data, because they reported not to have received the intervention, so in total 195 participants were analysed. Participants from the final sample were on average 58.7 years old $(\mathrm{SD}=13.5)$ and $67.2 \%$ were women.

Procedure and design. A pre-test-post-test control group design was used with the type of flyer (intervention, control) as independent variable, and fruit intake (FI) and vegetable intake (VI) as dependent variables. The participants were invited for the study via email and after the sign-up procedure was finished, participants received an email with the online pre-test. Next, the participants were randomly assigned to either the intervention condition $(N=95)$ or the control condition $(N=100)$. In the next three days, the researchers made phone calls to all participants with an explanation of the study, to guarantee that participants understood the procedure and to minimize non-response. The intervention materials were sent in envelopes with an extra letter with explanation. Ten days after the intervention was sent to the home environment, participants received an email with the post-test.

Intervention. Participants in the control condition received a flyer with positive outcomes of fruit and vegetable intake [3]. Participants in the intervention condition received a flyer with the same information as the control flyer, but with additional information in the form of multiple techniques that aimed to increase self-efficacy. Participants received the intervention materials at home, to capture real dietary behaviour. Participants were asked to read the flyer thoroughly. 
$\mathrm{BFJ}$

122,11

3300

Self-efficacy manipulation. We operationalized the multiple self-efficacy techniques as follows: one side of the intervention flyer contained a short text convincing that participants were capable of eating the recommended amount of fruit and vegetables (verbal persuasion; Bandura, 1977) and a short assignment in which participants had to recollect and write down an event in which they successfully ate fruit or vegetables instead of something unhealthy (performance accomplishments; Bandura, 1977). Two examples were given, to facilitate the recollection of a successful event. The other side of the intervention flyer contained a recipe (Meeusen et al., 2010). The recipe was introduced as easy to make, cheap, tasty and healthy and aimed to give participants an actual experience in which they successfully prepared and consumed a healthy meal (performance accomplishments; Bandura, 1977).

Measurements. Measurements included the same pre- and post-tests for both conditions. The dependent variables were FI and VI.

Fruit and vegetable intake. Fruit and vegetable intake was measured with the short Food Frequency Questionnaire validated in the Netherlands (Bogers et al., 2004). It measures the consumption of five categories of fruit (tangerines; citrus fruits; apples and pears; bananas; other fruit), two categories of vegetables (prepared vegetables; raw vegetables) and fruit and vegetable juice (which is categorized as fruit). For every fruit and vegetable category, there were two items: "how many days did you eat fruit/vegetable in the past week?" with the response options "not", "1 day", "2 days" ... "7 days" and "how many serving spoons/pieces/ glasses of fruit/vegetable did you eat in one day on average?" with the response options " 1 ", "1.5", " 2 " ... "5 or more". The analyses were done with the average amount of fruit and vegetable intake per day. This was calculated by multiplying the amount of days a week with the amount of times per day (which equals the total amount of fruit and vegetable intake in the past week), divided by 7 . The reproducibility ( 1 month: $r^{\text {veg }}=0.73, r^{\text {fruit }}=0.80 ; 1$ year: $r^{\text {veg }}=0.81, r^{\text {fruit }}=0.62$ ) and relative validity (positive modest correlations between fruit and vegetable intake and biomarkers and between changes in both measures) of these measures are good (Bogers et al., 2004).

Self-efficacy. A fruit and vegetable intake self-efficacy scale that was validated in a Dutch population was used to measure self-efficacy towards VI and self-efficacy towards FI (Bannink and van der Bijl, 2011). The questionnaire was initially validated amongst highschool students, but was adapted for this study to be suitable for adults (e.g. by deleting questions as "I am sure that I can eat fruit without my parents being there").

The VI self-efficacy scale (VSE-S) has 13 items and the FI self-efficacy scale (FSE-S) has 14 items. All items start with "I am sure that I. .." followed by vegetable items such as "can eat vegetables as a snack" and "can eat vegetables when I have to prepare it myself" and fruit items such as "can eat fruit during breakfast" and "can eat two pieces of fruit a day". Items were rated on a seven-point scale with the anchors "I surely cannot" (1) and "I surely can" (7). Mean scores for self-efficacy towards FI and towards VI were calculated for the data analysis.

A factor analysis revealed two dimensions of fruit self-efficacy (FSE) and two dimensions of vegetable self-efficacy (VSE). These two dimensions are fruit/vegetable consumption during a meal and fruit/vegetable consumption as a snack. This distinction between the types of self-efficacy (meal vs snack) was taken into consideration for the analyses. The measurements of the constructs are reliable $\left(\alpha^{\mathrm{FSE} \_ \text {snack }}=0.913 ; \alpha^{\mathrm{FSE} \_ \text {meal }}=0.814\right.$; $\alpha^{\mathrm{VSE} \_ \text {snack }}=0.912 ; \alpha^{\mathrm{VSE} \_ \text {meal }}=0.836$ ).

Intention. The "TasteNet-panel" used for the recruitment likely consists of people who are generally interested in food-related topics and who frequently participate in other food studies. Therefore, we measured the intention to eat fruit and vegetables after the intervention to check how motivated the participants were and to control for this in the analyses. Intention was measured with the items "how many pieces of fruit are you planning to eat every day in the coming week?" and "how many serving spoons of vegetables are you 
planning to eat every day in the coming week?" These were open-ended questions (Godinho et al., 2013) [4].

Outcome beliefs. As positive outcome beliefs are a prerequisite for the effectiveness of selfefficacy (Bandura, 1977), outcome beliefs were measured at post-test so we could control for this variable in the analyses. Outcome beliefs were measured with ten statements, rated on a seven-point scale with the anchors "I totally disagree" (1) and "I totally agree" (7). The statements started with "If I eat at least two pieces of fruit and $200 \mathrm{~g}$ of vegetables a day..." followed by items such as "then I will improve my health" and "then I will lose weight". The measurement of the construct is reliable $(\alpha=0.867)$, thus a mean score was calculated for the data analysis.

Demographics and practical checks. Finally, demographics were measured and various questions were added to the online questionnaire at post-test, to check if the implementation of the intervention was successful. We asked whether participants read the flyer, if they successfully completed the assignment on the flyer and if they prepared the recipe on the flyer.

Analyses. There are multiple methods to analyse pre-test-post-test control designs (with mediation). Van Breukelen (2006) recommends to use an ANCOVA in randomized studies with a pre-test-post-test control design. To test the mediated effect in the pre-test-post-test control group design, the ANCOVA model is also recommended (Valente and MacKinnon, 2017). Therefore, we decided to apply an ANCOVA to test the main effects and the mediation effects.

\section{Results}

Fruit and vegetable intake [5]. Fruit intake. An ANCOVA with flyer (intervention, control) as independent variable and FI at post-test as dependent variable, while controlling for FI at pretest, intention to eat fruit and outcome beliefs, was performed. Results revealed no main effect of flyer on FI $(F(1,184)=1.759 ; p=0.186 ; \eta[2]=0.009)$. Participants who received the intervention-flyer reported no different FI $(M=0.045)$ than participants who received the control-flyer $(M=0.045)$.

Vegetable intake. An ANCOVA with flyer (intervention, control) as independent variable and VI at post-test as dependent variable, while controlling for VI at pre-test, intention to eat fruit and outcome beliefs, was performed. Results revealed a marginally significant effect of flyer on VI $(F(1,187)=3.298 ; p=0.071 ; \eta[2]=0.017)$. Participants who received the intervention-flyer had a marginally significant higher VI $(M=1.97)$ than participants who received the control-flyer $(M=1.81)$.

The intervention-flyer marginally significantly increased self-reported VI and had no effect on self-reported FI.

The mediating role of self-efficacy concerning the flyer and fruit and vegetable intake. We performed a mediation analysis following the procedure of Hayes and Preacher (2014) and including the pre-test of the dependent variable, intention at post-test and outcome beliefs as covariates. The mediation was verified by using the bootstrap method with $95 \%$ confidence interval (CI). Results show that self-efficacy did not mediate the effect of the intervention-flyer on FI, and self-efficacy with regard to eating vegetables as a snack also did not mediate the effect of the intervention-flyer on VI. However, self-efficacy with regard to eating vegetables during a meal negatively mediated the effect of the intervention-flyer on VI.

Fruit intake. As can be seen in Figure 1, participants who received the intervention-flyer did not experience more self-efficacy with regard to eating fruit as a meal $\left(a^{M 1}=-0.176, p^{M 1}\right.$ $=0.207)$ or as a snack $\left(a^{M 2}=-0.159, p^{M 2}=0.115\right)$, and participants who experienced more self-efficacy did not report to eat more fruit $\left(b^{M 1}=0.005 ; p^{M 1}=.744 ; b^{M 2}=.004 ; p^{M 2}=.840\right)$. A bootstrap CI for the indirect effect $\left(a b^{M 1}=-0.001 ; a b^{M 2}=-0.001\right)$ based on 5,000

\section{Brief self- efficacy interventions}

3301 
$\mathrm{BFJ}$ 122,11

3302

Figure 1.

Simple mediation model for the influence of the intervention flyer on fruit intake through self-efficacy

Figure 2.

Simple mediation model for the influence of the intervention flyer on vegetable intake through self-efficacy bootstrap samples was not entirely above or below zero $\left(\mathrm{CI}^{M 1}\right.$ : -0.0102 to $0.0030 ; \mathrm{CI}^{M 2}$ : -0.0091 to 0.0044). In line with the results from the ANCOVA, there was also no evidence that participants who received the intervention flyer reported to eat more fruit $\left(c^{, M 1}=0.039\right.$; $p^{M 1}=0.193 ; c^{\prime M 2}=0.031 ; p^{M 2}=0.299$ ).

Vegetable intake. As can be seen in Figure 2, participants who received the interventionflyer experienced less self-efficacy with regard to eating vegetables as a meal $\left(a^{M 3}=-0.210\right.$; $\left.p^{M 3}=0.046\right)$ and as a snack $\left(a^{M 4}=-0.404 ; p^{M 4}=0.017\right)$. Participants who experienced more self-efficacy reported to eat (marginally) significantly more vegetables $\left(b^{M 3}=0.176\right.$; $\left.p^{M 3}=0.006 ; b^{M 4}=0.080 ; p^{M 4}=0.051\right)$. A bootstrap CI for the indirect effect $\left(a b^{M 3}=-0.037\right.$; $a b^{M 4}=-0.032$ ) based on 5,000 bootstrap samples was entirely below zero for self-efficacy with regard to eating vegetables during a meal $\left(\mathrm{CI}^{M 3}:-0.1040\right.$ to -0.0038$)$, however, not entirely below or above zero for self-efficacy with regard to eating vegetables as a snack $\left(\mathrm{CI}^{\mathrm{M4}}\right.$ : -0.0919 to 0.0007$)$. In line with the results from the ANCOVA, there was evidence that participants who received the intervention flyer reported to eat more vegetables $\left(c^{M 3}=0.195 ; p^{M 3}=0.035 ; c^{M 4}=0.193 ; p^{M 4}=0.041\right)$.

\section{Discussion study 1}

The results of the first study partly support our first hypothesis (H1) stating that a brief selfefficacy intervention increases fruit and vegetable intake. Respondents who received the intervention-flyer show an increase in self-reported VI after two weeks. However, we did not find evidence that the intervention-flyer showed an increase in self-reported FI. This might be

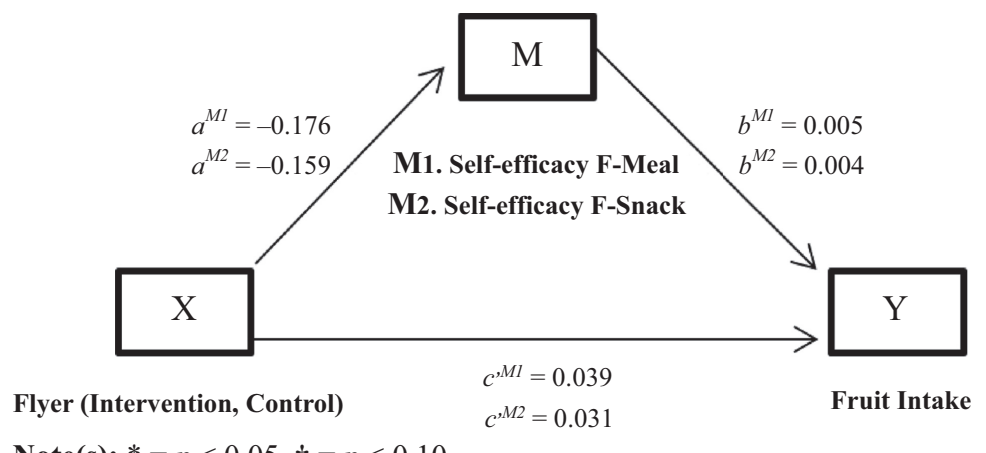

Note(s): $*=p<0.05, \dagger=p<0.10$

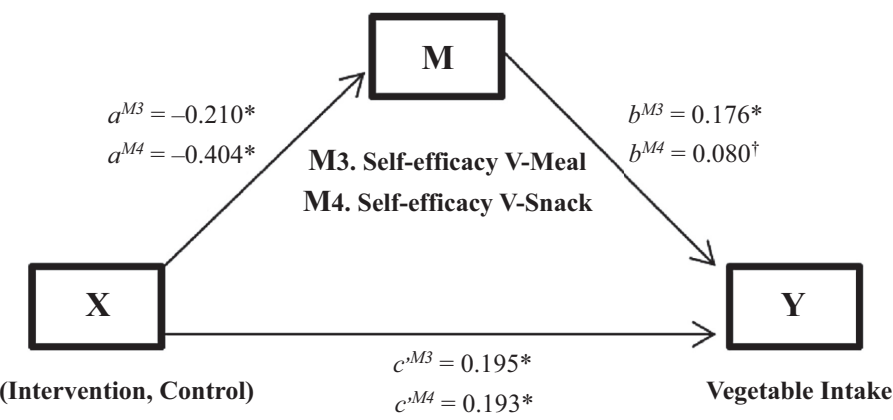

Flyer (Intervention, Control)

$c^{, M 4}=0.193^{*}$

Vegetable Intake

Note(s): ${ }^{*}=p<0.05, \dagger=p<0.10$ 
explained by the fact that the intervention-flyer focussed more on vegetables, because it included a recipe for a meal with vegetables.

The evidence for our second hypothesis (H2), stating that self-efficacy mediates the effect of a brief self-efficacy intervention on fruit and vegetable intake, is less straightforward. Our results indicate that self-efficacy with regard to eating vegetables during a meal negatively mediates the effect of the intervention-flyer on VI. However, we also found a positive direct effect of the intervention-flyer on VI and a positive relation between self-efficacy with regard to eating vegetables during a meal and VI. Thus, despite the negative effect of the intervention on self-efficacy, the intervention and self-efficacy are both positively associated with VI. Self-efficacy possibly decreased because participating in a dietary study can be an unpleasant reminder about failure to perform a challenging task (Pedersen et al., 2016). This inconsistent mediation model might be caused by the presence of a suppression effect (MacKinnon et al., 2000). A suppression variable is generally defined as "a variable which increases the predictive validity of another variable (or set of variables) by its inclusion in a regression equation" (Conger, 1974, pp. 36-37). This happens quite often in studies that find a significant indirect effect and a non-significant total effect (Loeys et al., 2015).

Summarizing, study 1 shows that a brief self-efficacy intervention successfully increased VI, but there is no straightforward, consistent mediation effect of self-efficacy. This can indicate that self-efficacy as a psychological process is difficult to manipulate in brief interventions (Spencer et al., 2005). Considering the intervention-flyer was a multi-component intervention implemented in the field, other psychological processes that were not measured might have influenced the effects found (Loeys et al., 2015). In study 2, we aim to get further insight into self-efficacy as a mediator in brief interventions by implementing a brief intervention in a more controlled setting (online) while manipulating one self-efficacy technique.

\section{Study 2 [6]}

Study 2 aimed to test whether a brief online single-component self-efficacy intervention can influence healthy dietary behaviour in the form of compliance with a plan to decrease either salt intake or fat intake or caloric intake. The self-efficacy technique was selected based on the meta-analysis from Prestwich et al. (2014) and aims to target performance accomplishments. It is a technique that can be effective without combining it with other techniques (Prestwich et al., 2014). To capture a broad range of healthy dietary behaviours, the outcome variable of study 2 focussed on decreasing unhealthy eating habits. Areas that can be improved on and that are familiar to most consumers are decreasing salt intake (Hendriksen et al., 2014), decreasing saturated fat intake (Eilander et al., 2015) and decreasing caloric intake (Crino et al., 2015). All participants made a diet plan focussing on one of these three areas and as an outcome variable we measure compliance to this diet plan.

\footnotetext{
Method

Participants. Data was collected by MSI-ACI, a specialized company that can blend different panel and sample sources for each study through one controlled platform. The sample of this study was representative for the Dutch population in terms of age, income, education, employment, family, culture and living conditions. This study also included an eligibility criterion, namely that participants had to be motivated to change their diet. Therefore, it was not essential to consider outcome beliefs and intentions as in study 1 . The following three screening questions were used: to what extent do you want to decrease your salt intake/ calorie intake/saturated fat intake? These questions could be answered on a seven-point scale $(1=$ completely not, 7 = completely). Participants were granted permission to enter the study
} 
BFJ

122,11

3304 and make a plan for one of these goals, when they scored a 5 or higher on at least one of these questions.

In total, 794 people participated at first measurement (T1) and 415 people participated in the second measurement (T2) [7]. For the analyses we included the 415 participants who participated in both measurements. Participants from the final sample were on average 49.8 years old $(\mathrm{SD}=16.5)$ and $58.4 \%$ were women. Gender, education level and level of dietary self-efficacy of the final sample were not statistically different compared to the dropouts. However, the mean age of the final sample $(M=49.8)$ was significantly higher than the mean age of the dropouts $(M=44.3 ; F(1,792)=21.983 ; p<0.01)$.

Procedure and design. At the first measurement (T1), participants filled out the questionnaire online and were randomly assigned to either the intervention condition $(N=398)$ or the control condition $(N=396)$. All participants made a plan to replace an unhealthy product with a healthy product [8]. The self-efficacy technique "performance accomplishments" was operationalized as follows: participants in the intervention condition read a short introductory text and were instructed to recollect a moment where they successfully made a good diet choice. Participants in the control condition were instructed to describe an average day in the week. After the manipulation, participants filled out control questions and demographics. One week after the intervention was communicated, the second measurement (T2) took place and participants filled out the questionnaire online. The dropout in the intervention condition was slightly higher (50\%) than in the control condition (45.5\%), resulting in 199 participants in the intervention condition and 216 participants in the control condition.

Measures. The dependent variables were compliance with the plan and future compliance with the plan, which were measured at post-test. Self-efficacy was measured at pre- and posttest. Demographics were measured at pre-test, and practical checks were measured at post-test.

Compliance with the plan. Compliance with the self-made diet plan was measured at posttest with two items. The items were: "To what extent did you execute your self-made plan?" and "How many times did you execute your self-made plan?" Future compliance was also measured at post-test with the following item: "To what extent are you planning to execute your self-made plan in the coming week?" All items were rated on a seven-point scale, where the first compliance item and the future compliance item had the anchors "totally not" (1) and "totally" (7), and the second compliance item had the anchors "rarely" (1) and "very often" (7). The two compliance items significantly correlated with each other (Pearson's $r=0.778$ ), thus for the data analysis a mean score was calculated.

Self-efficacy. The Eating Self-Efficacy Scale (ESES; Glynn and Ruderman, 1986; derived from Bandura, 2006) was used to measure self-efficacy towards dietary changes on T1 and T2. The scale has 25 items and all items start with "It is difficult to maintain a healthy diet. .." followed by items such as "during the holidays", "when I am irritated" and "when I am hungry". The scale was adapted to the Dutch language, through back-translation. Items were rated on a seven-point scale with the anchors "not difficult" (1) and "very difficult" (7). The measurement of the construct is reliable $(\alpha=0.965)$, thus a mean score was calculated for the data analysis.

Demographics and practical checks. Finally, demographics were measured and to check if the implementation of the self-efficacy intervention was successful, we asked whether participants in the intervention condition were able to think of a moment they were successful in making a good diet choice (yes, no) and how difficult they thought it was to remember such a moment on a seven-point scale with the anchors "easy" (1) and "difficult" (7).

Analyses. To measure the main effect of the self-efficacy manipulation on compliance and future compliance, an ANOVA sufficed. There was no need to control for outcome beliefs and 
intentions, because participants were screened beforehand on their motivation to change their diet. Similar to study 1, we applied an ANCOVA to test the mediation effects, where selfefficacy at pre-test served as a covariate.

\section{Results}

Compliance with the plan [9]. An ANOVA with self-efficacy (yes, no) as independent variable and compliance as dependent variable was performed. Results revealed no main effect of selfefficacy on compliance $(F(1,413)=0.000 ; p=0.988 ; \eta[2]=0.000)$. Participants who received the self-efficacy manipulation reported no different compliance $(M=4.39)$ than participants in the control group $(M=4.38)$.

An ANCOVA with self-efficacy (yes, no) as independent variable and future compliance as dependent variable was performed. Results revealed no main effect of self-efficacy on future compliance $(F(1,413)=0.012 ; p=0.915 ; \eta[2]=0.000)$. Participants who received the selfefficacy manipulation reported no different compliance $(M=5.06)$ than participants in the control group $(M=5.07)$.

The mediating role of self-efficacy. To test the indirect effects, we performed a mediation analysis following the procedure of Hayes and Preacher v.2.16.3. The mediation was verified by using the bootstrap method with 95\% CI. Results showed that the self-efficacy manipulation indirectly influenced compliance $(Y 1)$ through its effect on dietary self-efficacy. No mediation effect was found for future compliance $(Y 2)$.

As can be seen in Figure 3, participants who received the self-efficacy manipulation experienced more dietary self-efficacy than those who do not receive the self-efficacy manipulation $(a=0.276 ; p=0.014)$, and participants who experienced more dietary self-efficacy reported to have executed their diet plan more $\left(b^{Y 1}=0.187 ; p^{Y 1}=0.018\right)$. A bootstrap CI for the indirect effect $\left(a b^{Y 1}=0.052\right)$ based on 5,000 bootstrap samples was entirely above zero $\left(\mathrm{CI}^{Y 1}\right.$ : 0.0045 to 0.1492$)$. However, participants who experienced more dietary self-efficacy did not intend to execute their diet plan more in the future $\left(b^{Y 2}=0.044\right.$; $p=0.571)$. A bootstrap CI for the indirect effect $\left(a b^{Y 2}=0.012\right)$ based on 5,000 bootstrap samples was not entirely above or below zero $\left(\mathrm{CI}^{Y 2}:-0.0405\right.$ to 0.0820$)$. In line with the results from the ANOVA, there was neither evidence that participants who received the self-efficacy manipulation reported to have executed their diet plan more in the past week $\left(c^{, Y 1}=-0.016 ; p=0.931\right)$ nor that they intended to execute their plan more in the future $\left(c^{, Y 2}=-0.016 ; p=0.929\right)$.

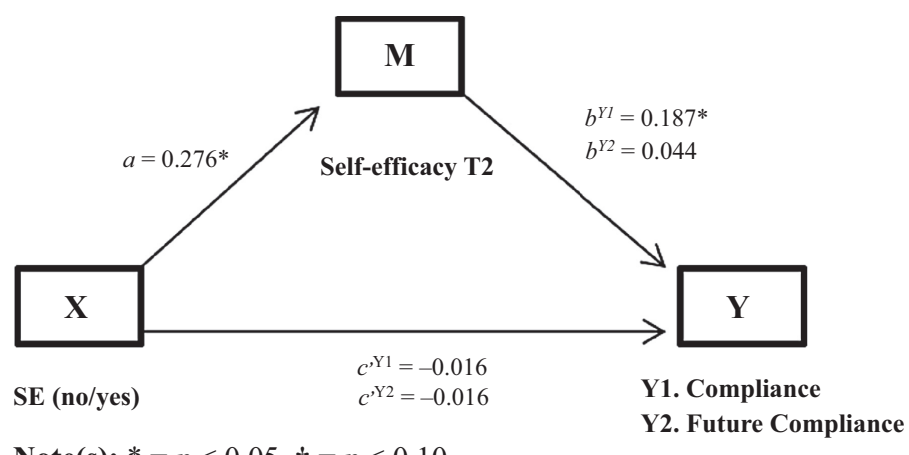

Note(s): $*=p<0.05, \dagger=p<0.10$

\section{Brief self- efficacy interventions}

3305
Figure 3. Simple mediation model for the influence of the self-efficacy technique on compliance and future compliance through dietary self-efficacy 
BFJ

122,11

3306

Discussion study 2

In study 2, we do not find evidence for our first hypothesis (H1). Results indicate that the brief self-efficacy intervention does not influence self-reported compliance with a diet plan or selfreported compliance with a diet plan in the future. However, we do find evidence for our second hypothesis (H2). Results show that participants who received the brief self-efficacy intervention increased their dietary self-efficacy and that participants with higher dietary self-efficacy also report to be more compliant with their diet plan. We did not find a similar mediation effect with future compliance.

Thus, in study 2, we find evidence that a brief self-efficacy intervention indirectly influenced compliance with a diet plan through self-efficacy, without the presence of a direct effect. The reason we find an indirect effect and not a direct effect can be explained by the fact that there is usually more power in the test of the indirect effect than in the test of the direct effect or total effect (Kenny and Judd, 2014). However, these results do need to be taken with caution, as Loeys et al. (2015) argue that considering indirect effects, in the absence of a total effect, can inflate the chances of a type I error.

\section{General discussion}

The aim of the current studies was to gain more insight into the effects of brief self-efficacy interventions on dietary behaviours and whether self-efficacy is the underlying mechanism that drives these effects. Brief interventions can be more cost-effective due to reduced time and expertise required for implementation compared with longer, multi-session interventions. Also, brief interventions have the potential to reach a larger and more diverse population, because they require less effort from participants in terms of time and commitment (Whatnall et al., 2018). In study 1, we found that a brief multi-component selfefficacy intervention, implemented at home, marginally significantly increased VI (bot not FI) compared to a control group. However, evidence for a positive mediation effect was absent, suggesting that self-efficacy was not necessarily the underlying mechanism for the effect on VI. In study 2, we found that a brief single-component self-efficacy intervention, implemented online, did not have a direct effect on healthy dietary behaviour in the form of reported compliance to eat healthier, but did indirectly increase the reported compliance through a mediation effect of dietary self-efficacy.

These findings bring forth multiple new insights concerning the concept of self-efficacy, brief interventions and mediation effects in field studies. Our results extend insights that in general brief interventions can affect dietary behaviours (Whatnall et al., 2018). Our studies showed that a brief intervention specifically targeted towards people's self-efficacy can directly increase VI and indirectly improve compliance to a diet plan to eat more healthily. We do not find an increase in FI, possibly because the brief intervention predominantly focussed on VI, as the intervention included a main meal recipe that contained vegetables, but not fruits.

A second aim was to gain more insight into the underlying mechanisms by which brief self-efficacy interventions influenced our outcomes, because this gives valuable information on the potential of interventions (O'Rourke and MacKinnon, 2018). Moreover, studying mediation is especially valuable when a concept is difficult to manipulate (Spencer et al., 2005), which seems to be the case with self-efficacy, as self-efficacy interventions show inconsistent results concerning the mediation of dietary self-efficacy. For instance, Luszczynska et al. (2007), Kreausukon et al. (2012) and Luszczynska et al. (2016) do find a mediation effect of dietary self-efficacy, while Guillaumie et al. (2012), Pedersen et al. (2016) and Keller et al. (2018) do not. In our studies we also find inconsistent mediation effects, namely that dietary self-efficacy does not - or negatively in the case of self-efficacy with regard to eating vegetables during a meal - mediate the effect of a multi-component brief 
self-efficacy intervention on fruit and vegetable intake, while it does fully mediate a singlecomponent brief self-efficacy intervention on compliance with a diet plan.

These studies provide more insight into the sensitivity of self-efficacy as a concept in brief dietary interventions and show that a different manipulation of self-efficacy can lead to different outcomes. For instance, we find that manipulating multiple self-efficacy techniques within a brief intervention is more effective to influence healthy dietary behaviours compared to manipulating one self-efficacy technique, which is line with the findings of Prestwich et al. (2014). This makes sense, as applying multiple techniques makes it likely that other mechanisms, besides self-efficacy, are (also) influenced that could have a positive effect on dietary behaviours. For example, in study 1, participants' self-identity could have been influenced by giving them a feeling of being someone who eats fruit and vegetables. Selfidentity as a healthy eater is found to be a direct driver of health behaviour (Strachan and Brawley, 2009; Carfora et al., 2016). Strachan and Brawley (2009) suggest that self-efficacy is an important factor for individuals to attain identity-behaviour congruence. The brief intervention was possibly helpful in matching one's identity with one's behaviour. Moreover, the brief self-efficacy intervention in study 1 could also have influenced attitudes. Information on the healthiness of fruit and vegetables could have created a more positive attitude towards fruits and vegetables. This in turn could have influenced intentions and behaviour following the theory of planned behaviour (TPB; Ajzen, 1991). Future research can test these potential other mechanisms that could also have been affected by our multi-component intervention.

Thus, the direct effect found in study 1 could be explained by other unmeasured mechanisms, besides self-efficacy, that were triggered by the brief intervention. That selfefficacy did not increase as result of the intervention can be explained by the fact that selfefficacy is a concept that is difficult to manipulate, as evidenced by the previously mentioned inconsistent findings regarding mediational effects of self-efficacy. Moreover, the effect of a self-efficacy intervention strongly depends on the self-efficacy technique that is used (Prestwich et al., 2014), and the extent to which dietary self-efficacy is influenced can differ between individuals (Keller et al., 2018). Part of the findings of study 1 even suggest that selfefficacy (regarding eating vegetables during a meal) negatively mediates the effect of the intervention on VI. An explanation can be that participating in a dietary study can negatively influence self-efficacy as it can be an unpleasant reminder about failure to perform a challenging task (Pedersen et al., 2016). Moreover, when testing an indirect effect, it remains possible that variables other than the independent variable influence both the mediator (selfefficacy) and the outcome variable, even though the independent variable is randomized (Loeys et al., 2015).

\section{Limitations and future research}

The current studies have some shortcomings, as well as avenues for future research. In both studies we used self-reported measures. Therefore, answering tendencies and biases might have affected the results, indicating the need for objective measures. However, measuring someone's diet objectively is a challenging task. There is a need for more objective outcome measures, in addition to self-reported outcome measures (Nour et al., 2016). It would be interesting to explore whether our findings agree with objective measurements of diet, for example, by looking at sales or receipts, using food diaries with pictures or skin measurements that can accurately measure fruit and vegetable intake (Mayne et al., 2013).

Additionally, in the current paper, it difficult to pinpoint what exactly caused the different findings in study 1 and study 2 , because the studies differed from each other on multiple aspects. Therefore, to gain a more systematic insight into the factors that determine the effects on dietary behaviours and whether dietary self-efficacy is mediating this effect, future studies could test brief self-efficacy interventions while only varying a certain set of aspects.

\section{Brief self- efficacy interventions}

3307 
BFJ 122,11
Moreover, to gain more insight into which other concepts play a role in dietary self-efficacy interventions, for example, self-identity, a measurement of these concepts could be considered.

\section{Conclusion}

Our studies provide first insights into the potential of brief self-efficacy interventions in improving dietary patterns. This can help practitioners apply less intensive and more costeffective techniques to improve healthy dietary behaviours on a broad scale. Moreover, our findings provide insight into the concept self-efficacy. Indication that self-efficacy can be influenced through brief techniques shows that it might not only be a concept that can be trained together with a professional but that it can also be activated from a distance without face-to-face contact.

Our findings also indicate that using multiple self-efficacy techniques in a brief intervention can have more impact on healthy dietary behaviours than using a single technique. Thus, when the aim is to reach impact in society, a multi-component approach can be the solution. However, using multiple techniques can give an unclear picture of the underlying mechanisms. Thus, when the goal is to gain more insight into these mechanisms, a single-component approach would be a better fit.

\section{Notes}

1. Food and Agriculture Organization of the United Nations

2. The study described is a 2 (point of choice prompt: yes, no) $x 2$ (flyer: intervention, control) betweensubjects design, with a pre-test and a post-test. We only describe the results from the self-efficacy manipulation, for it is relevant for this paper.

3. Positive outcomes of fruit and vegetable intake were communicated, because positive outcome beliefs are a prerequisite for self-efficacy to influence behaviour (Bandura, 1977). However, we only wanted to measure the effect of the self-efficacy manipulation, thus the positive outcomes of fruit and vegetable intake were mentioned on both flyers.

4. Two other items were used to measure intention, but these were not used in the analysis, because they were not valid. "I am planning to eat more vegetables in the coming week" and "I am planning to eat more fruit in the coming week".

5. Descriptive analysis shows that $99 \%$ of participants indicated to have read the flyer, $76 \%$ have prepared the recipe on the flyer and $59 \%$ have successfully made the assignment on the flyer. There were no significant differences on fruit and vegetable intake and self-efficacy between participants who made the recipe or the assignment compared to those who did not.

6. The study described is a 2 (implementation intention: yes, no) $x 2$ (self-efficacy: yes, no) $x 2$ (freedom of choice: yes, no) between-subjects design. We only describe the results from the self-efficacy manipulation, for it is relevant for this paper.

7. Due to budget reasons, recruitment stopped when about $50 \%$ of the participants were reached.

8. This study was part of a larger project. For reasons of conciseness, we only report part of the project. Some participants made more extensive plans than others, adding time and place to their plan (implementation intentions). The plans had drop-down menus, and the amount of choices participants received also varied (freedom of choice).

9. Descriptive analysis shows that $77 \%$ of the participants indicated that they were able to think of a moment where they were successful in making a good diet choice and on average this was experienced as fairly easy $(M=3.7 ; \mathrm{SD}=2.0$, on a seven-point scale with the anchors "easy" (1) and "difficult" (7)). There were no significant differences on compliance and self-efficacy between participants who were able to think of a moment where they were successful in making a good diet choice compared to those who did not. 


\section{References}

Ajzen, I. (1991), "The theory of planned behaviour", Organizational Behaviour and Human Decision Processes, Vol. 50, pp. 179-211.

Annesi, J.J. (2011), "Self-regulatory skills usage strengthens the relations of self-efficacy for improved eating, exercise, and weight in the severely obese: toward an explanatory model", Behavioural Medicine, Vol. 37 No. 3, pp. 71-76, doi: 10.1080/08964289.2011.579643.

Bandura, A (1977), "Self-efficacy: toward a unifying theory of behavioural change", Psychological Review, Vol. 84 No. 2, p. 191, doi: 10.1016/0146-6402(78)90002-4.

Bandura, A (2006), "Guide for constructing self-efficacy scales", in Pajares, F. and Urdan, T. (Eds), Self-efficacy Beliefs of Adolescents, Greenwich, CT: Information Age, pp. 307-336.

Bannink, R. and van der Bijl, J.J. (2011), "Reliability and validity of a fruit and vegetable self-efficacy instrument for secondary-school students in The Netherlands", Public Health Nutrition, Vol. 14 No. 5, pp. 817-825, doi: 10.1017/S1368980010003368.

Bogers, R.P., Van Assema, P., Kester, A.D., Westerterp, K.R. and Dagnelie, P.C. (2004), "Reproducibility, validity, and responsiveness to change of a short questionnaire for measuring fruit and vegetable intake", American Journal of Epidemiology, Vol. 159 No. 9, pp. 900-909, doi: 10.1093/ aje/kwh123.

Brink, L., Postma-Smeets, A., Stafleu, A. and Wolvers, D. (2016), Richtlijnen Schijf van Vijf, Stichting Voedingscentrum Nederland, Den Haag, 4e druk.

Carfora, V., Caso, D. and Conner, M. (2016), "The role of self-identity in predicting fruit and vegetable intake", Appetite, Vol. 106, pp. 23-29, doi: 10.1016/j.appet.2015.12.020.

Conger, A.J. (1974), "A revised definition for suppressor variables: a guide to their identification and interpretation”, Educational and Psychological Measurement, Vol. 34, pp. 35-46, doi: 10.1177/ 001316447403400105.

Crino, M., Sacks, G., Vandevijvere, S., Swinburn, B. and Neal, B. (2015), "The influence on population weight gain and obesity of the macronutrient composition and energy density of the food supply", Current Obesity Reports, Vol. 4 No. 1, pp. 1-10, doi: 10.1007/s13679-014-0134-7.

Eilander, A., Harika, R.K. and Zock, P.L. (2015), "Intake and sources of dietary fatty acids in Europe: are current population intakes of fats aligned with dietary recommendations?", European Journal of Lipid Science and Technology, Vol. 117 No. 9, pp. 1370-1377, doi: 10.1002/ejlt.201400513.

Fernández, B.R., Warner, L.M., Knoll, N., Montenegro, E.M. and Schwarzer, R. (2015), "Synergistic effects of social support and self-efficacy on dietary motivation predicting fruit and vegetable intake", Appetite, Vol. 87, pp. 330-335, doi: 10.1016/j.appet.2014.12.223.

Glynn, S.M. and Ruderman, A.J. (1986), "The development and validation of an eating self-efficacy scale", Cognitive Therapy and Research, Vol. 10 No. 4, pp. 403-420, doi: 10.1007/BF01173294.

Godinho, C.A., Alvarez, M.J. and Lima, M.L. (2013), "Formative research on HAPA model determinants for fruit and vegetable intake: target beliefs for audiences at different stages of change", Health Education Research, Vol. 28 No. 6, pp. 1014-1028, doi: 10.1093/her/cyt076.

Guillaumie, L., Godin, G. and Vezina Im, L.A. (2010), "Psychosocial determinants of fruit and vegetable intake in adult population: a systematic review", International Journal of Behavioural Nutrition and Physical Activity, Vol. 7, pp. 1-12, doi: 10.1186/1479-5868-7-12.

Guillaumie, L., Godin, G., Manderscheid, J.C., Spitz, E. and Muller, L. (2012), "The impact of selfefficacy and implementation intentions-based interventions on fruit and vegetable intake among adults", Psychology and Health, Vol. 27 No. 1, pp. 30-50, doi: 10.1080/08870446.2010.541910.

Hayes, A.F. and Preacher, K.J. (2014), "Statistical mediation analysis with a multicategorical independent variable", British Journal of Mathematical and Statistical Psychology, Vol. 67 No. 3, pp. 451-470, doi: 10.1111/bmsp.12028.

Hendriksen, M.A., van Raaij, J.M., Geleijnse, J.M., Wilson-van den Hooven, C. and Ocké, M.C. (2014), "Monitoring salt and iodine intakes in Dutch adults between 2006 and 2010 using 24 h urinary

\section{Brief self- efficacy interventions}

3309 
BFJ 122,11

sodium and iodine excretions", Public Health Nutrition, Vol. 17 No. 7, pp. 1431-1438, doi: 10. 1017/S1368980013001481.

Kellar, I. and Abraham, C. (2005), "Randomized controlled trial of a brief research-based intervention promoting fruit and vegetable consumption", British Journal of Health Psychology, Vol. 10 No. 4, pp. 543-558, doi: 10.1348/135910705X42940.

Keller, J., Motter, S., Motter, M. and Schwarzer, R. (2018), "Augmenting fruit and vegetable consumption by an online intervention: psychological mechanisms", Appetite, Vol. 120, pp. 348-355, doi: 10.1016/j.appet.2017.09.019.

Kenny, D.A. and Judd, C.M. (2014), "Power anomalies in testing mediation", Psychological Science, Vol. 25 No. 2, pp. 334-339, doi: 10.1177/0956797613502676.

Kreausukon, P., Gellert, P., Lippke, S. and Schwarzer, R. (2012), "Planning and self-efficacy can increase fruit and vegetable consumption: a randomized controlled trial", Journal of Behavioural Medicine, Vol. 35 No. 4, pp. 443-451, doi: 10.1007/s10865-011-9373-1.

Loeys, T., Moerkerke, B. and Vansteelandt, S. (2015), "A cautionary note on the power of the test for the indirect effect in mediation analysis", Frontiers in Psychology, Vol. 5, p. 1549, doi: 10.3389/ fpsyg.2014.01549.

Luszczynska, A., Tryburcy, M. and Schwarzer, R. (2007), "Improving fruit and vegetable consumption: a self-efficacy intervention compared with a combined self-efficacy and planning intervention", Health Education Research, Vol. 22 No. 5, pp. 630-638, doi: 10.1093/her/cyl133.

Luszczynska, A., Horodyska, K., Zarychta, K., Liszewska, N., Knoll, N. and Scholz, U. (2016), "Planning and self-efficacy interventions encouraging replacing energy-dense foods intake with fruit and vegetable: a longitudinal experimental study", Psychology \& Health, Vol. 31 No. 1, pp. 40-64, doi: 10.1080/08870446.2015.1070156.

Mayne, S.T., Cartmel, B., Scarmo, S., Jahns, L., Ermakov, I.V. and Gellermann, W. (2013), "Resonance Raman spectroscopic evaluation of skin carotenoids as a biomarker of carotenoid status for human studies", Archives of Biochemistry and Biophysics, Vol. 539 No. 2, pp. 163-170, doi: 10. 1016/j.abb.2013.06.007.

MacKinnon, D.P., Krull, J.L. and Lockwood, C.M. (2000), "Equivalence of the mediation, confounding and suppression effect”, Prevention Science, Vol. 1 No. 4, pp. 173-181.

Meeusen, M.J.G., Van Wijk, E.E.C., Hoogendam, K., Ronteltap, A. and Van 't Riet, J. (2010), Eetgewoonten Van Laagopgeleiden, LeiRapport, Den Haag.

Nour, M., Chen, J. and Allman-Farinelli, M. (2016), "Efficacy and external validity of electronic and mobile phone-based interventions promoting vegetable intake in young adults: systematic review and meta-analysis", Journal of Medical Internet Research, Vol. 18 No. 4, doi: 10.2196/ resprot.4665.

Obesity and overweight (2018), available at: https://www.who.int/news-room/fact-sheets/detail/obesityand-overweight/ (accessed 11 April 2019).

O'Rourke, H.P. and MacKinnon, D.P. (2018), "Reasons for testing mediation in the absence of an intervention effect: a research imperative in prevention and intervention research", Journal of Studies on Alcohol and Drugs, Vol. 79 No. 2, pp. 171-181, doi: 10.15288/jsad.2018.79.171.

Pedersen, S., Grønhøj, A. and Thøgersen, J. (2016), "Texting your way to healthier eating? Effects of participating in a feedback intervention using text messaging on adolescents' fruit and vegetable intake", Health Education Research, Vol. 31 No. 2, pp. 171-184, doi: 10.1093/her/ cyv104.

Prestwich, A., Kellar, I., Parker, R., MacRae, S., Learmonth, M., Sykes, B. and Castle, H. (2014), "How can self-efficacy be increased? Meta-analysis of dietary interventions", Health Psychology Review, Vol. 8 No. 3, pp. 270-285, doi: 10.1080/17437199.2013.813729.

Spencer, S.J., Zanna, M.P. and Fong, G.T. (2005), "Establishing a causal chain: why experiments are often more effective than mediational analyses in examining psychological processes", Journal of Personality and Social Psychology, Vol. 89 No. 6, p. 845, doi: 10.1037/0022-3514.89.6.845. 
Storm, V., Reinwand, D., Wienert, J., Kuhlmann, T., De Vries, H. and Lippke, S. (2017), "Brief report: compensatory health beliefs are negatively associated with intentions for regular fruit and vegetable consumption when self-efficacy is low", Journal of Health Psychology, Vol. 22 No. 8, pp. 1094-1100, doi: 10.1177/1359105315625358.

Strachan, S.M. and Brawley, L.R. (2009), "Healthy-eater identity and self-efficacy predict healthy eating behaviour: a prospective view", Journal of Health Psychology, Vol. 14 No. 5, pp. 684-695, 1094-1100. doi: 10.1177/1359105309104915.

Valente, M.J. and MacKinnon, D.P. (2017), "Comparing models of change to estimate the mediated effect in the pretest-posttest control group design", Structural Equation Modeling: A Multidisciplinary Journal, Vol. 24 No. 3, pp. 428-450, 1094-1100, doi: 10.1080/10705511.2016. 1274657.

Van Breukelen, G.J. (2006), "ANCOVA versus change from baseline had more power in randomized studies and more bias in nonrandomized studies", Journal of Clinical Epidemiology, Vol. 59 No. 9, pp. 920-925, doi: 10.1016/j.jclinepi.2006.02.007.

Whatnall, M.C., Patterson, A.J., Ashton, L.M. and Hutchesson, M.J. (2018), "Effectiveness of brief nutrition interventions on dietary behaviours in adults: a systematic review", Appetite, Vol. 120, pp. 335-347, 1094-1100, doi: 10.1016/j.appet.2017.09.017.

World Health Organisation (2003), Diet, Nutrition and the Prevention of Chronic Diseases: Report of a Joint WHO/FAO Expert Consultation, WHO Technical Report Series 916, World Health Organisation, Geneva.

\section{Web References}

http://statline.cbs.nl/StatWeb/publication/?VW=T\&DM=SLNL\&PA=37296ned\&D1 =a\&D2=0,10,20,30, 40,50,60,(1-1),1\&HD=130605-0924\&HDR=G1\&STB=T/ (accessed 11 April 2019).

Obesity and overweight (2018), available at: https:/www.who.int/news-room/fact-sheets/detail/ obesity-and-overweight/ (accessed 11 April 2019).

\section{Corresponding author}

Emily P. Bouwman can be contacted at: emily.bouwman@wur.nl

\section{Brief self- efficacy interventions}

3311 